\title{
Athermal Martensite Transformation Curve
}

\author{
Paulo Rangel Rios ${ }^{a *}$, José Roberto Costa Guimarães ${ }^{a, b}$ \\ ${ }^{a}$ Escola de Engenharia Industrial Metalúrgica de Volta Redonda, Universidade Federal Fluminense- \\ UFF, Av. dos Trabalhadores, 420, CEP 27255-125, Volta Redonda, RJ, Brasil \\ ${ }^{b}$ Mal. Moura 338H/22C, CEP 05641-000, São Paulo, SP, Brasil
}

Received: November 16, 2015; Revised: January 11, 2016; Accepted: February 16, 2016

\begin{abstract}
In a recent paper, the present authors [Materials Research. 2015. 18(3): 595-601] demonstrated that the thickness of athermal martensite plates had a thermally activated component. The reason for this was probably the interaction of the athermal glissile martensite-austenite interface with dislocations located within the surrounding austenite. Based on this result, here we derive a new expression for the volume fraction against temperature for athermal martensite transformation. This new expression includes a thermal activated term. The expression obtained here gives good agreement with athermal martensite transformation in $\mathrm{Fe}-\mathrm{Ni}-\mathrm{C}$ and $\mathrm{Fe}-\mathrm{C}$ alloys.
\end{abstract}

Keywords: Martensitic phase transformation, Microstructure, Analytical methods, steels

\section{Introduction}

The relevance of martensitic transformation in materials science and technology does not need reiteration. The close relationship of martensite to technology has existed since Osmond's pioneer work ${ }^{1}$. In this context, the rationalization of the martensite microstructure to the transformation curve continues to be of interest.

The current understanding is that martensite transformation is displacive with a defined crystallography. In the present case, we focus on athermal martensite. Athermal martensite transformation comprises two steps. The first step is the springing-up of martensite units. This step is "barrier-less". This means that a martensite unit starts to form without needing to overcome any kind barrier. Clearly, this step does not involve thermal activation. The second step is the growth of those units. This growth normally involves the migration of a glissile interface. This migration takes place without thermal activation. Nonetheless, a glissile interface may interact with dislocations that may exist in its path. Those interactions are dislocation processes that may produce a drag and hinder interface migration. Our previous work suggest that these interactions may result in a thermal activated term for growth. Therefore, a thermal activated component may appear during athermal martensite transformation. This may occur even if the springing-up of its units and interface migration are not themselves thermal activated. This paper proposes a new expression for volume fraction against transformation temperature of athermal martensite that captures this thermal activated component.

The typical microstructure resultant from martensitic transformation comprises a large number of units of decreasing $\operatorname{size}^{2,3}$, organized to minimize the shear component of the transformation strain $^{3}$. The mutual impingement of the

*e-mail: prrios@id.uff.br martensite units, as well as existing austenite obstacles impervious to the displacive mechanism of the transformation such as grain boundaries, delimit the size of the units. It is understood that martensite initially propagates from preferred austenitic defects (PADs), and proceeds by autocatalysis. After springing-up, a martensite unit does not continue to grow or coalesce. The rate of propagation of martensite is very high, which rules-out martensite growth by thermally activated atom transfer at the interface ${ }^{4}$. Instead, current understanding considers that the martensite transformation is nucleation-controlled. Nonetheless, thermally activated ("isothermal") and non-thermal activated ("athermal") aspects can be extracted from the transformation curves ${ }^{5-7}$, and the role of dislocation processes in the core of martensite transformation is presently consolidated ${ }^{8}$.

For athermal martensite one may think of a transformation curve to be a volume fraction, $V_{V}$, as a function of the transformation temperature, $T$, that is, $V_{V}(T)$. Empirical expressions of this kind are available for martensite transformation. The most well-known is the Koistinen-Marburger ${ }^{9}$

$V_{V}(T)=1-\exp \left(-\alpha\left(M_{S}-T\right)\right)$

where $M_{S}$ is the martensite start temperature and $\alpha$ is a positive constant.

Guimarães and $\operatorname{Rios}^{10}$ have recently derived a more fundamentally based expression, specifically for lath martensite

$V_{V}(T)=1-\exp \left(-\beta \frac{T_{M}-T}{T}\right)$

where $T_{M}$ is the highest temperature at which an embryo becomes viable. In practice, it is close to $M_{S}$ martensite start temperature. It is worthy of note the original approach taken by Shankaraiah et al., ${ }^{11-14}$ to simulate the martensite transformation by means of Monte Carlo simulation. 


\section{Model derivation}

Of special importance to the present work is the martensite transformation of $\mathrm{Fe}-30 \mathrm{wt} \% \mathrm{Ni}$ particulate reported by $\mathrm{Cech}$ and Turnbull ${ }^{15}$ that convincingly demonstrated martensite's heterogeneous aspect. Furthermore, their results indicate that the preferred austenite defects (PADs) for martensite nucleation are scarce and randomly distributed. Therefore, the probability of finding a particle with at least one such sites $^{16}$ is

$$
P(n, T)=1-\exp \left(-q n_{V}^{T}\right)
$$

where $q$ is the mean particle volume and $n_{V}^{T}$ is the number density of the PADs at temperature $T$.

We conceive that the propagation of a martensite unit from one PAD yields other plates by autocatalysis which instantaneously propagate and that these units are located within the austenite volume were they formed. Thence, the number of units generated by one PAD, including intergrain spread, is equal to $a_{C}$. Admitting the extended transformation concept advanced by JMAK ${ }^{17-19}$, the extended number density of martensite units generated by $n_{V}^{T}$ PADs is

$$
N_{V}=a_{C} n_{V}^{T}
$$

As a consequence, the (extended) volume fraction of martensite is $N_{V} v_{M}=v_{M} a_{C} n_{V}^{T}$, where $v_{M}$ is the mean volume of a martensite plate supposing that its growth is not hindered by other plates, in other words, $v_{M}$ is an extended martensite mean volume. Therefore, the extended fraction transformed, $V_{V E}$, is $V_{V E}=v_{M} a_{C} n_{V}^{T}$. Furthermore, one admits that the classical relationship between volume fraction and extended volume fraction ${ }^{17-21}, V_{V}=1-\exp -V_{V E}$, applies here. As a result at a temperature, $T$

$V_{V}(T)=1-\exp \left(-v_{M} a_{C} n_{V}^{T}\right)$

Moreover, the present authors ${ }^{22}$ reviewing the data from Cech and Turnbull ${ }^{15}$ found out that $n_{V}^{T}$ could be fitted to the equation

$$
n_{V}^{T}=n_{V}^{0} \frac{\Delta S}{k}\left(\frac{T_{M}-T}{T}\right)
$$

with a high fitting correlation. In this equation $n_{V}^{0}$ is the general number density of PADs , $\Delta S$ is the transformation entropy change, $k$ is the Boltzmann constant, and $T_{M} \geq M_{S}$ is the temperature at which those sites become operational. $M_{S}$ is the martensite start temperature.

In a recent work ${ }^{23}$, the present authors examined in detail the temperature dependence of the mean "intrinsic" (or extended) dimensions of the martensite units in Fe-31wt $\%$ Ni-0.02wt $\% \mathrm{C}$. The conclusions were that the diameter, $D$, remained roughly temperature independent and scaled with the grain size (the martensite partitions the austenite grains) whereas the thickness, $t$, decreased with temperature with an Arrhenius like temperature dependence

$t=t_{0} \exp \left(-E_{A} / k T\right)$

where the activation energy, $E_{A}$, is about $1.2 \times 10^{-20} \mathrm{~J} /$ event for the Fe-Ni-C alloy. The present authors attributed this temperature dependence not to a thermally activated martensite growth but rather to a thermally activated arrest of growth owing to the interaction of the moving martensite interface with the surrounding austenite plasticity.

In this paper, considering that martensite extended thickness is thermally activate ${ }^{23}$ we propose that the martensite extended volume, $v_{M}=\pi t D^{2} / 4$, is also thermally activated

$v_{M}=v_{M}^{0} \exp \left(-E_{A} / k T\right)$

Combining the equations above

$V_{V}(T)=1-\exp \left(-A\left(\frac{T_{M}-T}{T}\right) \exp -\frac{E_{A}}{k T}\right)$

This is the athermal martensite transformation curve where the pre-factor, $A$, is $A=v_{M}^{0} n_{V}^{0} a_{C} \frac{\Delta S}{k}$.

\section{Experimental data}

This work mainly refers to Fe-Ni-C alloys in the range of $30-33 w t \% \mathrm{Ni}$ and low carbon $(\leq 0.02 \mathrm{wt} \%)$. These alloys were used to investigate different aspects of the martensite transformation ${ }^{3,5,24-29}$ that have sub-zero transformation. Thence, these alloys are amenable to metallographic analysis at room temperature to characterize the progress of the transformation at different temperatures. Our reference material is $\mathrm{Fe}-31 \mathrm{wt} \% \mathrm{Ni}-0.02 \mathrm{wt} \% \mathrm{C}^{30-32}$. Complemental, we considered for comparison the data independently obtained typical of Fe-1.86wt $\% \mathrm{C}^{33}$ and of $\mathrm{Fe}-32 \mathrm{wt} \% \mathrm{Ni}-0.04 \mathrm{wt} \%$ alloys $^{5}$. The values of these microstructure descriptors used in the present work were taken from the referenced publications, by scanning and digitizing the relevant figures, which, unfortunately, did not include error bars. However, our familiarity with the methodology permits ascribing $\pm 10 \%$ to volume fraction figures obtained by systematic procedures on a planar random section of the specimen. Notwithstanding that, note that the handling of each material is described in the referenced papers ${ }^{5,30-33}$ and are not repeated here for brevity sake. Figs. 1 and 2 show typical micrographs of a Fe-Ni-C alloy partially transformed and almost fully transformed to martensite.

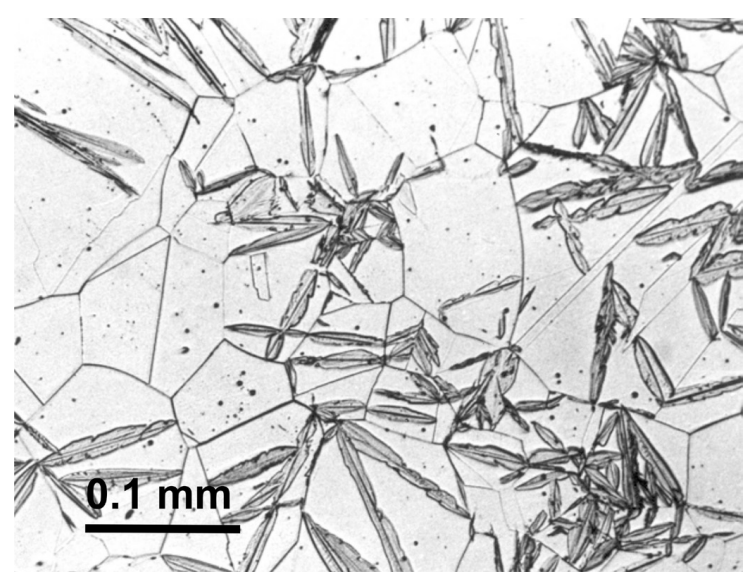

Fig. 1 - Light micrograph showing the microstructure of a coarse-grained austenite of a Fe-31wt $\% \mathrm{Ni}-0.02 \mathrm{wt} \% \mathrm{C}$ alloy transformed at $M_{S}=220 \mathrm{~K}$. (Courtesy of J. R. C. Guimarães) 


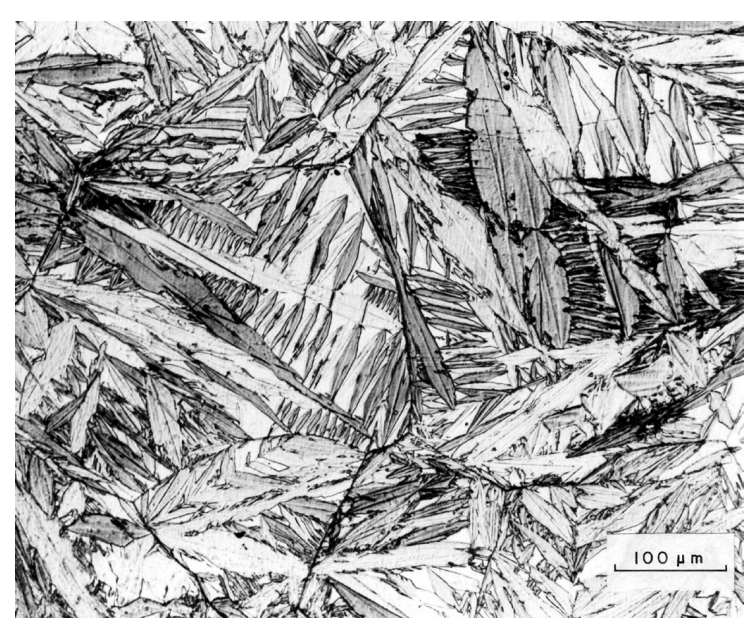

Fig. 2 - Light micrograph showing the microstructure of a coarse-grained austenite of a Fe-31wt $\% \mathrm{Ni}-0.02 \mathrm{wt} \% \mathrm{C}$ alloy transformed by cooling into liquid nitrogen. (Courtesy of J. R. C. Guimarães)

\section{Results and discussion}

Figures 3-9 show a plot of $V_{V}$ against transformation temperature data obtained for the $\mathrm{Fe}-\mathrm{Ni}-\mathrm{C}$ alloys ${ }^{5,30}$ and for the $\mathrm{Fe}-\mathrm{C}$ alloy ${ }^{33}$. In figures 3-9 the data show error bars. These error bars are not in the original papers and they were drawn estimating the relative error of the volume fraction measurements to be $\pm 10 \%$.

A nonlinear regression of the model, equation 9, to the $\mathrm{Fe}-\mathrm{Ni}-\mathrm{C}$ and $\mathrm{Fe}-\mathrm{C}$ data gave high coefficients of determination, $\mathrm{R}^{2}$, better than 0.93 in all cases except for Fe-Ni-C with $0.049 \mathrm{~mm}$ of reference ${ }^{30}$ in which $\mathrm{R}^{2}=0.78$. Therefore, in all cases the model, equation 9, exhibited good agreement with the experimental data.

It is important to examine the parameters obtained by the fitting. For Fe-Ni-C alloys $T_{M}$ ranged from 221 to $233 \mathrm{~K}$ whereas experimental $M_{S}$ ranged from 212 to $226 \mathrm{~K}$. The mean of fitted $T_{M}$ parameters was $227 \mathrm{~K}$ whereas the mean $M_{S}$ was $221 \mathrm{~K}$. Considering that $T_{M}$ is the temperature at which the first martensite propagation sites (PADs) become viable, it should be higher but comparable to $M_{S}$ that is the temperature in which the start of martensite transformation was detected. $T_{M}$ is a theoretical concept as it has been experimentally impossible to identify such propagation sites whereas $M_{S}$ is an experimental concept and the detection of martensite start depends on the sensitivity of the technique used. Even using sophisticated the techniques, it is clear that a certain number of propagation sites (PADs) must have already generated martensite units in order for detection to be possible. Hence, $T_{M} \geq M_{S}$ even though one would expect this difference to be small and $6 \mathrm{~K}$ looks like a reasonable value. For the Fe-C alloy $^{33} T_{M}$ was equal to $320 \mathrm{~K}$. Mendiratta and Krauss did not measure $M_{S}$ but obtained it by extrapolation of $M_{S}$ from lower C contents. From that extrapolation, they considered $M_{S}$ about $323 \mathrm{~K}$ that is consistent with the value of the fitted $T_{M}$ parameter. Therefore, broadly speaking, although $T_{M}$ was obtained by fitting it gave a very good agreement with experimental values.

Another critical parameter is the activation energy, $E_{A}$. The mean value of the fitted $E_{A}$ values was $E_{A} \cong 10^{-20} \mathrm{~J} /$ event that is in good agreement with the value obtained in our

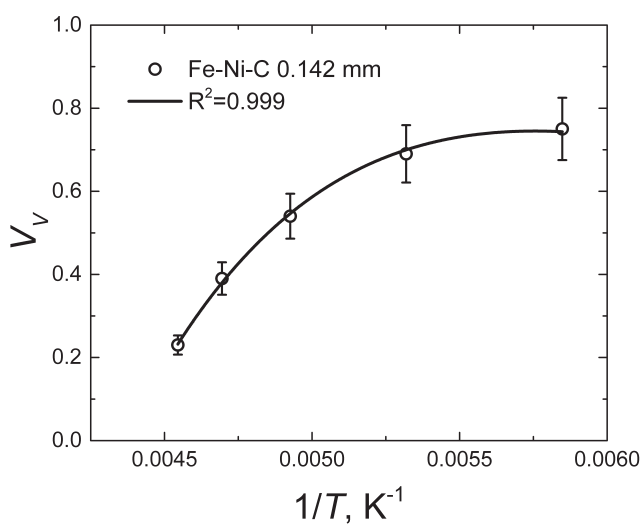

Fig. 3 - Volume fraction of martensite plates per unit of volume against transformation temperature. Fe-31wt $\% \mathrm{Ni}-0.02 \mathrm{wt} \% \mathrm{C}$ alloy ${ }^{30}$ with a mean intercept length of austenite grains equal to $0.142 \mathrm{~mm}$. Solid line corresponds to the model developed here: equation 9. Error bars corresponding to an estimated relative error of $\pm 10 \%$ were added to the plot.

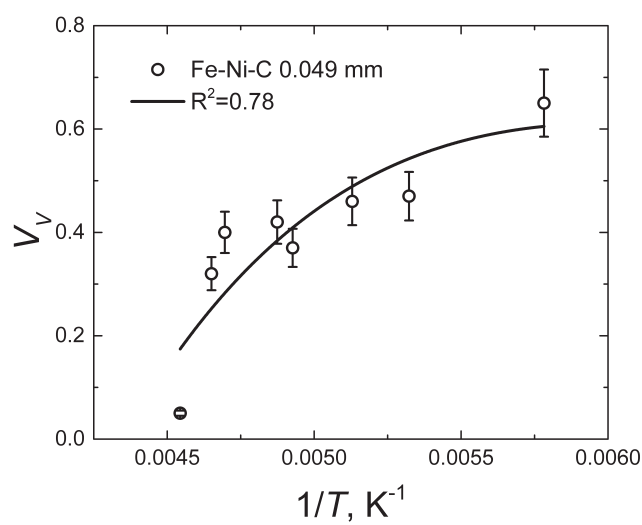

Fig. 4 - Volume fraction of martensite plates per unit of volume against transformation temperature. Fe-31wt $\% \mathrm{Ni}-0.02 \mathrm{wt} \% \mathrm{C}$ alloy ${ }^{30}$ with a mean intercept length of austenite grains equal to $0.049 \mathrm{~mm}$. Solid line corresponds to the model developed here: equation 9. Error bars corresponding to an estimated relative error of $\pm 10 \%$ were added to the plot.

previous work $\mathrm{k}^{23}$ and compares with the activation energy for dislocation processes in austenite ${ }^{23}$ for a detailed discussion of this.

Finally, we acknowledge that it is not possible to make a detailed analysis of the fitted parameter $A=v_{M}^{0} n_{V}^{0} a_{C} \frac{\Delta S}{k}$. It is apparent that $A$ is a lump factor composed of factors that are not easy to estimate.

Nevertheless, expeditiously taking $a_{C} \approx 10^{3} \mathrm{after}^{34}$, admitting that $v_{M}^{0}$ is a fraction $m(0.01-0.1)$ of the mean austenite grain volume which is reasonable in the case of the Fe-Ni-C alloys, and ${ }^{35} \frac{\Delta S}{k}=0.55$, we estimate the average $n_{V}^{0}$ value in the range $1.2-1.6 \times 10^{4} \mathrm{~mm}^{-3}$ which compares with the $\mathrm{e}^{23} 5.4 \times 10^{4} \mathrm{~mm}^{-3}$ typical of the particulate material described by Cech and Turnbull ${ }^{15}$.

Henceforth, we can conclude that the present model gives good agreement with Fe-Ni-C alloys and a Fe-C alloy over a 


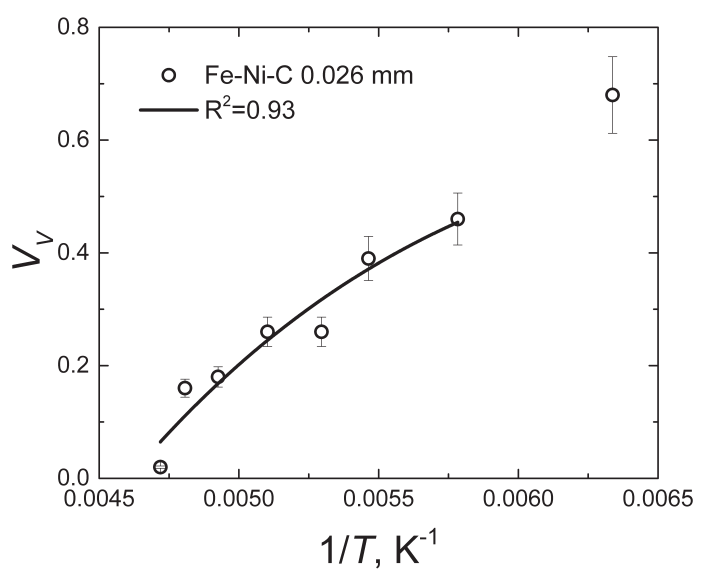

Fig. 5 - Volume fraction of martensite plates per unit of volume against transformation temperature. Fe-31wt $\% \mathrm{Ni}-0.02 \mathrm{wt} \% \mathrm{C}$ alloy ${ }^{30}$ with a mean intercept length of austenite grains equal to $0.026 \mathrm{~mm}$. Solid line corresponds to the model developed here: equation 9. Error bars corresponding to an estimated relative error of $\pm 10 \%$ were added to the plot. Outlier was excluded from the fitting.

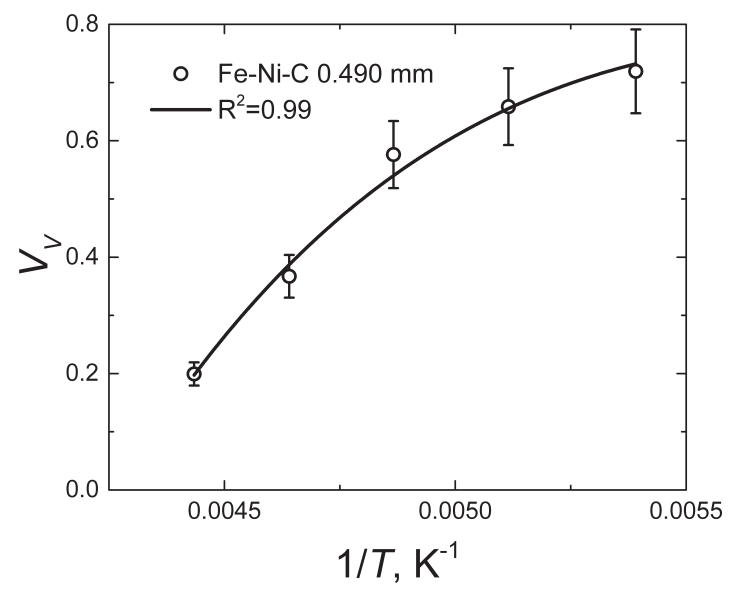

Fig. 6 - Volume fraction of martensite plates per unit of volume against transformation temperature. Fe-32wt $\% \mathrm{Ni}-0.004 \mathrm{wt} \% \mathrm{C}$ alloy ${ }^{5}$ with a mean intercept length of austenite grains equal to $0.490 \mathrm{~mm}$. Solid line corresponds to the model developed here: equation 9. Error bars corresponding to an estimated relative error of $\pm 10 \%$ were added to the plot.

broad range of grain sizes, from $0.026 \mathrm{~mm}$ up to $0.490 \mathrm{~mm}$. Even though at small grain sizes such as 0.026 one observes the presence of martensite spread ${ }^{36}$.

Noteworthy, small values of $E_{A} / k T$ yields

$V_{V}=1-\exp \left(-A \frac{T_{M}-T}{T}\right)$

This simplification is applicable in two cases. At high temperatures where austenite plasticity is not an issue such as in case of lath martensite transformation in low-medium carbon steels ${ }^{10}$. In addition, if the mechanical autocatalysis/variant selection suffices to accommodate the transformation strains, one may assume that $E_{A} \approx 0$. Equation 9 is formally identical to equation 2 .

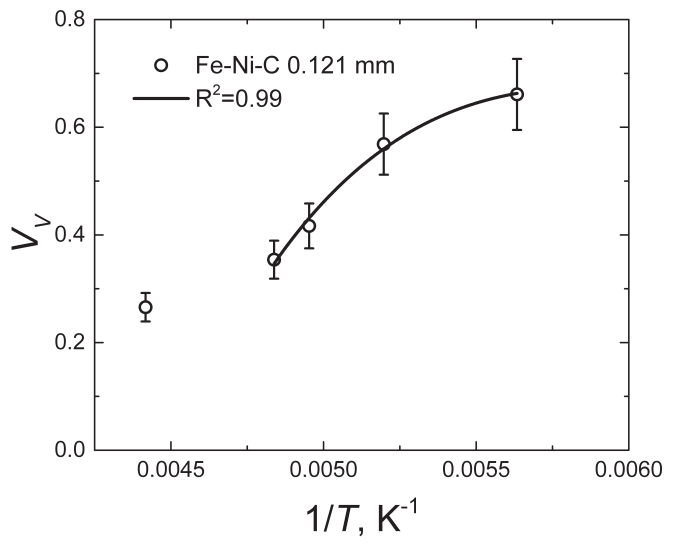

Fig. 7 - Volume fraction of martensite plates per unit of volume against transformation temperature. Fe-32wt $\% \mathrm{Ni}-0.004 \mathrm{wt} \% \mathrm{C}$ alloy with a mean intercept length of austenite grains equal to $0.121 \mathrm{~mm}$. Solid line corresponds to the model developed here: equation 9. Error bars corresponding to an estimated relative error of $\pm 10 \%$ were added to the plot. Outlier was excluded from the fitting.

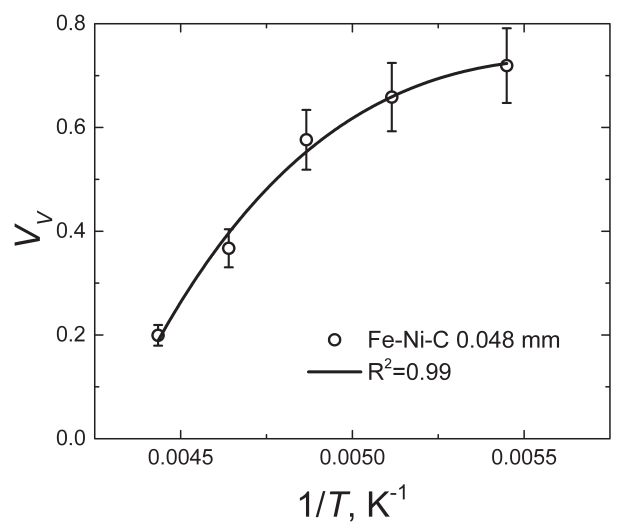

Fig. 8 - Volume fraction of martensite plates per unit of volume against transformation temperature. Fe-32wt $\% \mathrm{Ni}-0.004 \mathrm{wt} \% \mathrm{C}$ alloy ${ }^{5}$ with a mean intercept length of austenite grains equal to $0.048 \mathrm{~mm}$. Solid line corresponds to the model developed here: equation 9. Error bars corresponding to an estimated relative error of $\pm 10 \%$ were added to the plot.

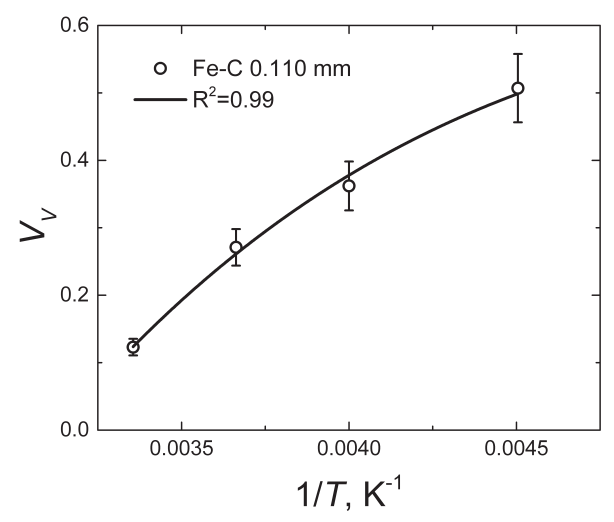

Fig. 9 - Volume fraction of martensite plates per unit of volume against transformation temperature. $\mathrm{Fe}-1.86 \mathrm{wt} \% \mathrm{C}$ alloy ${ }^{30}$ with a mean intercept length of austenite grains equal to $0.110 \mathrm{~mm}$. Solid line corresponds to the model developed here: equation 9. Error bars corresponding to an estimated relative error of $\pm 10 \%$ were added to the plot. 


\section{Summary and conclusions}

We propose a new model of volume fraction of athermal martensite as a function of transformation temperature. The model includes a thermal activated martensite plate volume. This is supported by the previous finding ${ }^{23}$ of a thermal activated martensite thickness. We attributed this thermal activated term to the drag effect of the austenite plasticity on the mobility of the martensite-austenite interface. The dislocations introduced in the adjacent austenite to assist in the relaxation of the transformation strains interact with the mobile athermal martensite-austenite interface. Notice that whereas dislocation processes in the austenite are thermally activated processes, the martensite-austenite interface movement itself is not regarded to be thermally activated $^{23}$. The comparison of the model, equation 9 with experimental data permits the following conclusions:

- In all cases examined here, Fe-Ni-C alloys and a Fe-C alloy, the model, equation 9 , exhibited good agreement with the experimental data.

\section{References}

1- Osmond MF. Méthode génerale pour l'analyse micrographique des aciers au carbone. Bulletin de la Société d'Encouragement pour Industrie Nationale. 1895;10(5):480-509.

2- Fisher JC, Hollomon JH, Turnbull D. Kinetics of the austenite -martensite transformation. Journal of Metals, Transactions Section. 1949;185: 691-700.

3- Bokros JC, Parker ER. Mechanism of martensite burst transformation in Fe-Ni single crystals. Acta Metallurgica. 1963;11(12):1291-1301.

4- Bunshah RF, Mehl RF. Rate of propagation of martensite. Transactions AIME. 1953; 197:1251-1258.

5- Lin M, Olson GB, Cohen M. Distribution-activation kinetics of heterogeneous martensitic nucleation. Metallurgical Transactions A. 1992;23(11):2987-2997.

6- Guimarães JR, Rios PR. Driving force and thermal activation in martensite kinetics. Metallurgical and Materials Transactions A. 2009;40(10):2255-2509.

7- Laughlin DE, Jones NJ, Schwartz AJ, Massalski TB. Thermally activated martensite: its relationship to non-thermally activated athermal martensite. In: Olson GB, Lieberman DS, Saxena A, editors. International Conference on Martensitic Transformation (ICOMAT). Carnegie Institute of Technology. p.141-144. http:// repository.cmu.edu/cgi/viewcontent.cgi?article $=1019 \&$ context $=$ mse

8- Olson GB, Cohen M. Principles of martensitic transformation. In: Meyers MA, Inal OT, editors. Frontiers in Materials Technologies. Amsterdam: Elsevier; 1985. p. 43-87.

9- Koistinen DP, Marburger RE. A general equation prescribing the extent of the austenite-martensite transformation in pure iron-carbon alloys and plain carbon steels. Acta Metallurgica. 1959;7(1):59-60.

10- Guimarães JR, Rios PR. Modeling lath martensite transformation curve. Metallurgical and Materials Transactions A. 2013;44(1):2-4.

11- Shankaraiah N, Murthy KP, Lookman T, Shenoy SR. Reequilibration after quenches in athermal martensites: conversion delays for vapor-to-liquid domain-wall phases. Physical Review B. 2015;91(6):214108.
- The parameter $T_{M}$ is the temperature at which the first embryo becomes viable. In the Fe-Ni-C alloys, the fitted value of the parameter $T_{M}$ is comparable to $M_{S}$. Here it was found $T_{M}$ to be on average $6 \mathrm{~K}$ above $M_{S}$.

- Moreover, under certain circumstances discussed in the text, our model, equation 9, may be simplified yielding a simpler expression than equation 9 , namely, equation 10. Equation 10 is formally identical to equation 2 previously obtained by the authors for lath martensite.

- The relaxation of the martensite transformation strains influences the size of the martensite units, tantamount the fraction transformed and the transformation curve of the alloys considered here.

\section{Acknowledgements}

P. R. Rios is grateful to Conselho Nacional de Desenvolvimento Científico e Tecnológico, CNPq, and to Fundação de Amparo à Pesquisa do Estado do Rio de Janeiro, FAPERJ, for financial support.

12- Shankaraiah N, Murthy KP, Lookman T, Shenoy SR. Athermal martensites, temperature-time-transformation diagrams and thermal hysteresis: Monte Carlo simulations of strain pseudospins. Solid State Phenomena. 2012;185(2):31-33.

13- Shankaraiah N, Murthy KP, Lookman T, Shenoy SR. Monte Carlo simulations of strain pseudospins: ahermal martensites, incubation times, and entropy barriers. Physical Review B. 2011;84(6):064119. DOI:http://dx.doi.org/10.1103/ PhysRevB.84.064119

14- Shankaraiah N, Murthy KP, Lookman T, Shenoy SR. Incubation times and entropy barriers in martensitic kinetics: Monte Carlo quench simulations of strain pseudospins. Europhysics Letters. 2010;92(11):36002, 1-6.

15- Cech RE, Turnbull D. Heterogeneous nucleation of the martensite transformation. Transactions AIME. 1956;206(2):124-132.

16- Cohen M, Olson GB. Martensite nucleation and the role of the nucleating defect. In: First JIM International Symposium on New Aspects of Martensitic Transformation, 1976 may 1012. Japan; Japan Institute of Metals. Materials Transactions. 1976;17:93-98.

17- Avrami MJ. Kinetics of phase change I general theory. The Journal of Chemical Physics. 1939;7(12):1103-1112.

18- Johnson WA, Mehl RF. Reaction kinetics in processes of nucleation and growth. Transactions AIME. 1939;135:416-441.

19- Kolmogorov NA. On the statistics theory of the crystallization of metals. Isvestiia Academii Nauk SSSR - Seriia Matematicheskaia. 1937;1:333-359

20- Rios PR, Villa E. Transformation kinetics for inhomogeneous nucleation. Acta Materialia. 2009;57(4):1199-1208.

21- Chiu SN, Stoyan D, Kendall WS, Mecke J. Stochastic Geometry and its Applications. 3rd ed. UK: John Willey \& Sons; 2013.

22- Guimarães JR, Rios PR. Initial nucleation kinetics of martensite transformation. Journal of Materials Science. 2008;43(15):52065210 .

23- Guimarães JR, Rios PR. Microstructural path analysis of martensite dimensions in $\mathrm{FeNiC}$ and $\mathrm{FeC}$ alloys. Materials Research. 2015;18(3):595-601. 
24- Machlin ES, Cohen M. Burst phenomenon in the martensitic transformation. Transactions AIME. 1951;191(9):746-754.

25- Zhang W, Jin YM, Khachaturyan AG. Phase field microelasticity modeling of heterogeneous nucleation and growth in martensitic alloys. Acta Materialia. 2007;55(2): 565-574.

26- Shibata A, Morito S, Furuhara T, Maki T. Substructures of lenticular martensites with different martensite start temperatures in ferrous alloys. Acta Materialia. 2009;57(2): 483-492.

27- Shibata A, Furuhara T, Maki T. Interphase boundary structure and accommodation mechanism of lenticular martensite in Fe-Ni alloys. Acta Materialia. 2010;58(9):3477-3492.

28- Heo TW, Chen LQ. Phase-field modeling of displacive phase transformations inelastically anisotropic and inhomogeneous polycrystals. Acta Materialia, 2014; 76(1):68-81.

29- Malik A. Phase change with stress effects and flow. [Thesis]. Stockholm: The Royal Institute of Technology; 2013. http:// kth.diva-portal.org/smash/get/diva2:606230/FULLTEXT01. pdf
30- Guimarães JR, Gomes JC. Metallographic study of influence of austenite grain-size on martensite kinetics in Fe-31.9 Ni-0.02C. Acta Metallurgica. 1978;26(10):1591-1596.

31- Guimarães JR, Gomes JC. Microstructural aspects of martensite transformation in coarse-grained Fe-31.1 pct Ni-0.02 pct C. Metallurgical and Materials Transactions A. 1979; 10(1):109-112.

32- Guimarães JR, Brandão LP. Determination of number and size-distribution of martensitic plates in Fe-31.9-percent-Ni0.02-percent-C. Scripta Metallurgica. 1980;14(3):305-310.

33- Mendiratta MG, Krauss G. Development of martensitic microstructure and microcracking in an Fe-1.86C alloy. Metallurgical Transactions A. 1972;3(7):1755-1760.

34- Guimarães JR, Saavedra A. A Computer-assisted analysis of the spread of martensite transformation. Materials Science Engineering. 1984;62(1):11-15.

35- Kaufman L Cohen M. Martensitic transformations. Progress in Metal Physics. 1958;7: 165-246.

36- Rios PR, Guimarães JR. Microstructural path analysis of athermal martensite. Scripta Materialia. 2007;57(12):1105-1108. 